\title{
Aspartic acid as a crystal growth catalyst.
}

\author{
S. Piana, F. Jones and J. D. Gale* \\ * Corresponding author \\ Nanochemistry Research Institute, Department of Applied Chemistry, Curtin University of Technology, \\ GPO Box U1987, Perth 6845, Western Australia. \\ Phone: +6189266 3838 Fax: +61892664699 \\ email: julian@ivec.org
}

\begin{abstract}
Ion desolvation is an important kinetic step in the growth of divalent ionic crystals - a category that encompasses numerous materials relevant to biomineralization. It has recently been shown for one such divalent ionic crystal that the rate-limiting desolvation of the cation can be assisted by the anion and that this process can be surface specific. Here we show that even a simple biological molecule, such as aspartic acid, can have a measurable catalytic effect on barite crystal growth and that this effect is related to the lowering of the activation barrier for cation desolvation. We therefore suggest that growth rate enhancement on specific faces through catalysis of the cation desolvation step may be a viable mechanism for the positive control of biomineralization.
\end{abstract}

Keywords barium sulfate, aspartic acid, crystal growth, biomineralization, ion desolvation, molecular dynamics simulation, scanning electron microscopy.

\section{Introduction}

Crystallisation is one of the most widespread and important reactions in chemistry and biology. However, the atomistic details of this process are still not fully understood for the majority of materials, especially for more complex crystal growth environments, such as that which occurs during biomineralization. A fundamental comprehension of exactly how nature can achieve highly specific control of morphology under ambient conditions is still elusive. 
In principle, inorganic materials can be grown to yield desired phases and shapes, either by promoting the growth of certain polymorphs and particular directions (positive control), or by inhibiting the growth of competing phases and facets (negative control). While there is abundant literature concerning the negative control by biological and synthetic molecules at high and low concentration ${ }^{1}$, the literature concerning positive control is comparatively scarce. The most well-known and widespread positive control of nucleation is by seeding of the desired phase. Interestingly, it has been demonstrated that nacre-specific proteins may promote, rather than inhibit, calcite crystal growth ${ }^{2,3}$ and a recent paper has shown that low concentrations of peptides containing aspartic acid can enhance the growth rate of calcite and that this effect is more pronounced for the peptide sequences containing larger amounts of aspartic acid ${ }^{4}$.

Barite sediments are of considerable importance for paleoenvironmental studies ${ }^{5-7}$ and barite precipitation from seawater can be actively promoted by living organisms such as foraminifera despite the seawater being undersaturated with respect to barite ${ }^{8}$. Barium sulfate can also be found biomineralized in various xenophyophorea (particle containing organisms) ${ }^{9}$ and even in catacomb wall bacterial films ${ }^{10}$. Biomimetic approaches based on growth on Langmuir monolayers ${ }^{11,12}$ have shown that carboxylate containing organics appear to alter the kinetic of barite precipitation rather than the overall thermodynamics. In a recent paper we have investigated the basic mechanisms of the growth barite from aqueous solution and found that cation desolvation is an important kinetic step for crystal growth and that this process can be efficiently catalyzed by the sulphate anion on specific faces ${ }^{13}$. This study raises the question of whether a biological carboxylate, such as aspartic acid, may catalyse crystal growth by a similar mechanism. While moderate to high concentrations of aspartic and polyaspartic acids have a strong inhibitory effect on the nucleation and growth of barite crystals ${ }^{14-16}$, the effect of very low concentrations of aspartic acid on the growth of barite is currently unknown. Here we have simulated the effect of low concentrations of aspartic acid on the cation desolvation step, which is ratelimiting for barite growth on the (001) face at low supersaturations ${ }^{13}$. From these calculations, it is observed that aspartic acid is able to rather efficiently assist the cation desolvation. Thus, at low concentrations and low supersaturations, it may actually promote, rather than inhibit, growth of the (001) crystal face. This prediction has been 
subsequently validated by experiments. Taken together, these results strongly suggest that positive control of crystal growth through catalytic ion desolvation is indeed a viable mechanism for biomineralization. 


\section{Methods}

\section{Materials}

Analytical reagent grade $\mathrm{Na}_{2} \mathrm{SO}_{4}$ and $\mathrm{BaCl}_{2}$ were both obtained from $\mathrm{BDH}$. Ultrapure water (18 megaohm, TOC $<0.01$ ) is used in all experiments. All the solutions were filtered through a $0.2 \mu \mathrm{m}$ membrane prior to use.

\section{Conductivity procedure}

The method adopted to measure conductivity for in-situ monitoring of barite precipitation has been discussed in detail elsewhere ${ }^{17,18}$. Briefly, a known amount of barium chloride is equilibrated at a given temperature to give the desired final $S^{*}$ value. Here the supersaturation ratio, $S^{*}$, is defined as $S^{*}=c / c_{e q}$, where $c$ is the solution concentration and $c_{e q}$ is the equilibrium value of $c$. Subsequently, aspartic acid was added, if required, and equilibrated. Although this should be corrected for the formation of the BaAsp complex, the $\log$ equilibrium constant for the $\mathrm{Ba}^{2+}+\mathrm{Asp}^{2-}=$ BaAsp complexation reaction is $1.95^{19}$. At the concentrations used in this work, the concentration of the complex is negligible $\left(<10^{-7} \mathrm{M}\right.$ at the highest barium and aspartic acid concentrations). Finally, a stoichiometric quantity of $\mathrm{Na}_{2} \mathrm{SO}_{4}$ solution was added to commence the precipitation reaction. The system is mixed with a glass stirrer and the conductivity probe measures the loss of mobile ions during the reaction (which, in the case of barium sulfate, is due to the reaction of barium ions with sulfate ions to produce barite). At the end of the run, the solids were filtered using a $0.2 \mu \mathrm{m}$ membrane, washed and dried in a dessicator prior to preparation for examination under a scanning electron microscope (SEM). The desupersaturation rate is calculated as the slope of the linear section of the conductivity curve after the induction period.

\section{SEM procedure}

A filter paper portion obtained from the crystallisation experiments was placed onto a carbon-coated SEM stub. Carbon paint was applied to ensure electrical contact and gold sputtered before viewing in a Philips XL30 SEM.

\section{Turbidity}


Precipitation conditions for turbidity measurements were the same as per the conductivity runs, except that the stirring speed was greater (300 rpm) to ensure a representative sample was brought close to the instrument window. Measurements were taken with an Analite NEP 160 from McVan Instruments with a $90^{\circ}$ detector. A reading was automatically logged to computer every 2 seconds and all runs were performed in duplicate. The data was subsequently averaged and smoothed for analysis.

\section{Molecular dynamics simulations}

Molecular dynamics simulations of the adsorption of $\mathrm{Ba}^{2+}$ on the (001) face of barite were performed using the program GROMACS ${ }^{20}$ using the force field parameters developed in a previous work ${ }^{13}$. Here the (001) surface is chosen for study based on the observation that it possesses the highest activation barrier to barium addition of the three surfaces previously examined ${ }^{13}$.

The starting structure was generated using the crystallographic unit cell of barite as a template. The program GDIS ${ }^{21}$ was used to generate a $6 \times 42 \mathrm{D}$ periodic surface. The surface was 4 layers deep and was transformed into a 3D periodic system by extending the cell axis in the $z$ direction. Both a barium and a sulphate ion, as well as an aspartic acid molecule, were added at $0.8 \mathrm{~nm}$ from the crystal surface. The empty space in the simulation cell was filled with 2266 SPC water molecules ${ }^{22}$. All bond lengths were constrained to their equilibrium positions with the LINCS algorithm ${ }^{23}$.

Non-bonded interactions were evaluated with a cutoff of $1.2 \mathrm{~nm}$. The Particle Mesh Ewald method $^{24}$ was used to treat long-range electrostatic interactions. The timestep of the simulations was 2 fs, with the non-bonded pair list being updated every 5 steps. Constant temperature simulations at $298.15 \mathrm{~K}$ were performed by coupling the system to a Nose-Hoover thermostat ${ }^{25}$ with a relaxation time of 3.0 ps. Constant pressure was obtained by coupling to an anisotropic Berendsen barostat ${ }^{26}$ with relaxation time of 5.0 ps. The compressibility of the system was set to $4.510^{-5} \mathrm{bar}^{-1}$ in the $z$ direction and 5.0 $10^{-6} \mathrm{bar}^{-1}$ in the $x$ and $y$ directions. The system was equilibrated by performing $100 \mathrm{ps}$ of NVT simulation at $298.15 \mathrm{~K}$ followed by 2 ns of NPT simulation at $298.15 \mathrm{~K}$ and $1 \mathrm{~atm}$.

\section{Umbrella sampling simulation}


An umbrella sampling simulation was performed where the distance between a $\mathrm{Ba}^{2+}$ ion and the crystal surface was progressively reduced from 0.6 to $0.1 \mathrm{~nm}$. Ten simulations were conducted where the position of the barium ion was constrained at distances ranging between 0.1 and $0.6 \mathrm{~nm}$ from the crystal surface. The force on the constraint was $3000 \mathrm{~kJ}$ $\mathrm{mol}^{-1} \mathrm{~nm}^{-1}$ and $1.6 \mathrm{~ns}$ of constrained MD simulation was performed for each point with the last 1.2 ns of each simulation used for data analysis.

\section{Results and Discussion}

Simulation of barium ion adsorption in the presence of aspartic acid

Previous studies have shown that cation desolvation is an important kinetic step for the growth and dissolution of barite ${ }^{27}$. This desolvation step can be assisted by the sulphate anion on the (010) and (210) faces, but not on the (001) crystal face ${ }^{13}$. As a consequence, at low supersaturation $\left(S^{*}<5\right)$ this is the slowest growing face of barite and its rate of growth is dominated by two-dimensional nucleation ${ }^{28}$. This raises the issue of whether a different ion may assist desolvation on the (001) face more efficiently than the sulphate ion and therefore promote crystal growth. In the context of biomineralization and in light of previous studies performed on calcite ${ }^{4}$ the effect of aspartic acid on the desolvation kinetic is of particular interest. Molecular dynamics simulations with the umbrella sampling methodology were carried out to study the adsorption of barium ions on the (001) face of barite in the presence of a single aspartic acid molecule. During the equilibration phase of the unconstrained simulation, the water molecules form a tightly bound layer on the surface of the barite crystal, in agreement with the X-ray reflectivity experiments $^{29}$. Previous studies have shown that permeation of ions through this double layer is an activated process ${ }^{13,30,31}$ and that permeation of anions is easier than permeation of cations ${ }^{13}$. It is found that aspartic acid spontaneously adsorbs on the crystal surface even during the equilibration period (Figure 1a), while the barium ion remains in solution. This is consistent with the experimental data that suggest that aspartic acid has a high affinity for the barite surface leading to the observed inhibition of barite at moderate concentrations $^{14-16}$.

As the $\mathrm{Ba}^{2+}$ ion approaches the surface, during the umbrella sampling simulation, it spontaneously forms an ion pair with the aspartic acid (Figure 1b). Due to its intrinsic 
flexibility, the aspartic acid molecule is able to coordinate the barium ion all the way down to the crystal surface (Figure 1c).

The data obtained from the umbrella sampling simulations were analyzed with the weighted histogram method ${ }^{32}$ to calculate reaction free energies. The activation energy barrier calculated in the presence of aspartic acid was compared with the barriers obtained in a previous work ${ }^{13}$ for an aspartic acid free environment (Figure 2). It is found that the activation energy barrier for bringing a barium ion to the crystal (001) surface is dramatically reduced by the aspartic acid interaction, dropping from 18 to $4 \mathrm{~kJ} \mathrm{~mol}^{-1}$. This effect is much larger than the effect of a sulphate ion adsorbed on the surface, which, for the (001) surface, only reduces the barrier to $14 \mathrm{~kJ} \mathrm{~mol}^{-1}$ (Figure 2). This result suggests that, while at moderate to high concentrations aspartic acid inhibits barite growth due to its high affinity for the barite surface, at low concentrations and low supersaturation it may actually act as a catalyst and promote the growth of the slow growing (001) face.

\section{Crystallization kinetics in the presence of aspartic acid}

The above hypothesis was tested by monitoring the barite precipitation kinetics in the presence of aspartic acid through conductivity measurements. Ideally, one would like to study the growth kinetics at the lowest possible supersaturation $\left(S^{*}<5\right)$ where the growth of the (001) face is rate limiting. Unfortunately, due to the instrument sensitivity it is not possible to obtain meaningful kinetic data at this supersaturation. For this reason, the crystal growth kinetics have been studied at a supersaturation ratio of 12.5 , where the rate of growth of the (001) face is no longer limiting, but still contributes to the overall growth rate.

Figure 3 illustrates that at aspartic acid concentrations of 2.5-4 $\mu \mathrm{M}$ the rate of desupersaturation increases by $40-50 \%$ as compared with the control run. Although there is considerable scatter amongst individual measurements, the effect is greater than the statistical uncertainty in the data. At higher concentrations the inhibitory effect of aspartic acid on crystal growth becomes dominant and the rate of desupersaturation begins to drop. This increase in the desupersaturation rate may be either due to an increase in the rate of nucleation or due to an increase in the rate of growth, or a combination of both. 
The rate of nucleation in the presence of aspartic acid was investigated with turbidity. Turbidity measurements were used to provide an estimate of the induction time. In all samples the turbidity remains almost constant up to $\sim 800 \mathrm{~s}$ (induction time) and then starts to grow linearly with time. In this range of concentrations, aspartic acid does not affect nucleation, as indicated by the same induction time measured for all the solutions, but may affect the growth kinetics. The rate of change of the turbidity after the induction period is influenced by the aspartic acid concentration. However, it is not trivial to directly relate it to a particle growth rate since the distribution of particles shapes and sizes also contributes to the signal ${ }^{33}$ (Figure 4). It is therefore concluded that enhanced crystal growth, rather that nucleation, should be responsible for the increase in the desupersaturation rate.

The morphology of particles grown at supersaturation ratios of 5 and 12.5 in the presence of aspartic acid was examined with Scanning Electron Microscopy and the aspect ratio between the $c$ and the $a$ axis of the crystals was measured (Figure 5). Note that the value in Figure 5 for zero concentration of aspartic acid represents aspect ratio for the control.

The measurements show that the overall morphology is preserved in the presence of $\mu \mathrm{M}$ concentrations of aspartic acid (Figure 6). However, at low supersaturation $\left(\mathrm{S}^{*}=5\right.$ ), crystals grown in the presence of $2.5 \mu \mathrm{M}$ aspartic acid have an aspect ratio three times larger than the control. This indicates that, at this supersaturation, aspartic acid is able to significantly enhance the growth rate of the (001) face relative to the other morphologically important faces. At $\mathrm{S}^{*}=12.5$ the effect is smaller and crystals grown in the presence of 2.5-4 $\mathrm{MM}$ aspartic acid are only $10-15 \%$ longer than crystals grown at either higher or lower concentrations (Figure 5). This result is completely consistent with the suggestion that aspartic acid can promote the cation desolvation. This kinetic step is expected to be rate limiting for the growth of the (001) face at low supersaturation and these are the conditions where the largest effect is observed. At higher supersaturation growth of the (001) face is no longer rate limiting. In this case the change in aspect ratio is only $10-15 \%$, while in the same conditions the enhancement in the desupersaturation ratio is $40-50 \%$ (Figure 3). This result may suggest that at higher supersaturations the effect of aspartic acid is less surface-specific or that other mechanisms may also 
contribute to the increase in the desupersaturation rate. Finally, it is observed that in most of the samples examined, the aspect ratio of the barite crystals has a binodal distribution, with a few crystals exhibiting a longer aspect ratio than the average (Figure 6). This effect is more pronounced for barite crystals grown at lower supersaturations. We speculate that this may be the result of the presence of screw dislocations on the (001) face that enhance the growth of some of the crystals.

\section{Conclusions}

Umbrella sampling simulations suggest that low concentrations of aspartic acid may have a catalytic effect on the growth of the (001) face of barite. This theoretical prediction has been validated by conductivity, turbidity and SEM experiments. These show:

i) that an increase in the rate of desupersaturation results at low concentrations of aspartic acid;

ii) that the effect on nucleation is negligible, and

iii) that the relative rate of growth of the (001) face in the presence of aspartic acid is faster than either in the control run or at aspartic acid concentrations where the net effect is inhibition of crystal growth.

These results point out that even a simple biological molecule, such as aspartic acid, may have a catalytic effect on crystal growth and that the effect can exhibit some surface specificity. Molecular dynamics simulations show that the catalytic effect is due to a decrease of the activation barrier for cation desolvation by the adsorbed aspartic acid molecules. As the growth of most divalent ionic solids follow remarkably similar kinetics $^{34}$, it is suggested that a similar mechanism is also effective for the observed promotion of calcite growth by aspartic acid containing peptides ${ }^{2-4}$ and therefore that catalysis of ion desolvation on specific faces by biological macromolecules may be a viable mechanism for the positive control of biomineralization.

\section{Acknowledgements}

We gratefully acknowledge the support of the Australian Government's Cooperative Research Centre (CRC) Program, through the Parker CRC for Integrated Hydrometallurgy Solutions for support of FJ, the Australian Research Council for a 
Fellowship for SP, the Government of Western Australia for a Premier's Research Fellowship for JDG, in addition to support for the Western Australian Nanochemistry Research Institute under the State Centres of Excellence programme, and iVEC and APAC for the provision of computing resources. 


\section{References}

1. $\quad$ Xu, A.-W.; Ma, Y.; Cölfen, H. J Mater Chem 2006, 17, 415-449.

2. $\quad$ Fu, G.; Qiu, S.; Orme, C.; Morse, D.; De Yoreo, J. Adv Materials 2005, 17, 2678.

3. $\quad$ Kim, I.; Darragh, M.; Orme, C.; Evans, J. Cryst Growth Des 2006, 6, 5-10.

4. $\quad$ Elhadj, S.; De Yoreo, J.; Hoyer, J.; Dove, P. Proc Natl Acad Sci U S A 2006, 103, 19237-19242.

5. $\quad$ Kastner, M. Proc Natl Acad Sci U S A 1999, 96, 3380-3387.

6. $\quad$ Turchyn, A.; Schrag, D. Science 2004, 303, 2004-2007.

7. $\quad$ Paytan, A.; Kastner, M.; Campbell, D.; Thiemens, M. Science 1998, 282, 14591462.

8. Bertram, M.; Cowen, J. Aquatic Geochem. 1998, 4, 455-468.

9. Hopwood, J. D.; Mann, S.; Gooday, A. J Marine Biol Ass UK 1997, 77, 969-987.

10. Sanchez-Moral, S.; Luque, L.; Canaveras, J.; Laiz, L.; Jurado, V.; Hermosin, B.;

Saiz-Jimenez, C. Annals of Microbiology 2004, 54, 1-12.

11. Heywood, B. R.; Mann, S. J Am Chem Soc 1992, 114, 4681-4686.

12. Heywood, B. R.; Mann, S. Langmuir 1992, 8, 1492-1498.

13. Piana, S.; Jones, F.; Gale, J. D. J Am Chem Soc 2006, 128, 13568-13574.

14. Jones, F.; Mocerino, M.; Ogden, M. I.; Oliveira, A.; Parkinson, G. M. Cryst Growth Des 2005, 5, 2336-2343.

15. $\quad$ Ross, R. J.; Low, K. C.; Shannon, J. E. Mater Performance 1997, 36, 53-57.

16. Collins, I. R. J Colloid \& Interface Sci 1999, 212, 535-544.

17. Jones, F.; Clegg, A.; Oliveira, A.; Rohl, A. L.; Ogden, M. I.; Parkinson, G. M.; Fogg, A. M.; Reyhani, M. M. CrystEngComm 2001, 40.

18. Jones, F.; Richmond, W. R.; Rohl, A. L. J Phys Chem B 2006, 110, 7414-7424.

19. May, P.; Murray, K. J Chem Eng data 2001, 46, 1035-1040.

20. Lindhal, E.; Hess, B.; van der Spoel, D. J Mol Mod 2001, 7, 306-317.

21. Fleming, S. D.; Rohl, A. L. Z Krist 2005, 220, (5-6), 580-584.

22. Berendsen, H. J. C.; Postma, J. P. M.; van Gunsteren, W. F.; Hermans, J.; Pullman, B., Intermolecular forces. Reidel: Dordrecht, The Netherlands, 1981; p 331342.

23. Hess, B.; Bekker, H.; Berendsen, H. J. C.; Fraaije, G. E. M. J Comp Chem 1997, 18, 1463-1472.

24. Essman, U.; Perera, L.; Berkowitz, M. L.; Darden, T. A.; Lee, H.; Pedersen, L. G. J Chem Phys 1995, 103, 8577-8593.

25. Nose, S. J Chem Phys 1984, 81, 511.

26. Berendsen, H. J. C.; Postma, J. P. M.; Van Gusteren, W. F.; DiNola, A.; Haak, J. R. J Chem Phys 1984, 81, 3684-3690.

27. Dove, P. M.; Czank, C. A. Geochim Cosmochim Acta 1995, 59, (10), 1907-1915.

28. Pina, C. M.; Becker, U.; Risthaus, P.; Bosbach, D.; Putnis, A. Nature 1998, 395, 483-486.

29. Fenter, P.; McBride, M. T.; Srajer, G.; Sturchio, N. C.; Bosbach, D. J Phys Chem B 2001, 105, 8112-8119.

30. Nielsen, A. E. J Cryst Growth 1984, 67, 289-310.

31. Kerisit, S.; Parker, S. C. J Am Chem Soc 2004, 126, 10152-10161. 
32. Roux, B. Comp Phys Comm 1995, 91, 275-282.

33. Gabas, N.; Hiquily, N.; Laguerie, C. Part Part Syst Charact 1994, 11, 121-126.

34. Nielsen, A. E.; Toft, J. M. J Cryst Growth 1984, 67, 278-288. 


\section{Figure captions}

Figure 1 Mechanism of $\mathrm{Ba}^{2+}$ adsorption in the presence of aspartic acid. Water molecules are shown as grey spheres, hydrogen atoms are not shown for clarity. a) Crystal surface after equilibration showing the ordered first hydration layer; b) At the beginning of the simulation the aspartic acid spontaneously coordinates the $\mathrm{Ba}^{2+}$ ion; c) the aspartic acid molecule assists the $\mathrm{Ba}^{2+}$ permeation through the water layer; d) at the end of the simulation the $\mathrm{Ba}^{2+}$ ion is on the surface, weakly coordinated by the aspartic acid. In b, c and $\mathrm{d}$ only the waters of the $\mathrm{Ba}^{2+}$ first hydration shell are shown.

Figure 2. Reaction free energy calculated from the umbrella sampling simulation for the (001) crystal face of barite. The free energy was calculated for a clean surface (red line), in the presence of a sulphate ion (green line) and in the presence of aspartic acid (blue line).

Figure 3 Relative de-supersaturation rates of barite precipitation in the presence of increasing aspartic acid concentration as measured by conductivity. The average control de-supersaturation rate is taken as a reference. Three independent measurements were performed for each data point.

Figure 4. Characterization of barite nucleation in the presence of aspartic acid using turbidity data as a function of time.

Figure 5. $c / a$ aspect ratio of barite crystals grown at $S^{*}=5$ (blue) and $S^{*}=12.5$ (red) in the presence of aspartic acid. Each data point is the result of 6 to 50 measurements and the vertical bar indicates the range of the distribution of measured values. Inset: a barite crystal showing the $c$ and $a$ axes.

Figure 6. Barite crystals morphology in the presence of increasing aspartic acid concentrations: a) $0 \mu \mathrm{M}$; b) $0.75 \mu \mathrm{M}$; c) $1.8 \mu \mathrm{M}$; d) $2.5 \mu \mathrm{M}$. All scale bars are $20 \mu \mathrm{m}$, except for a), which is $5 \mu \mathrm{m}$. Crystals were grown at a supersaturation $\mathrm{S}^{*}=5$. One of the outliers observed in the $0.75 \mu \mathrm{M}$ sample is indicated with a red circle. 
